\title{
Caracterisation Lithostratigraphique et Sedimentologique des Depôts des Sondages de Fresco et de Grand-Lahou (Sud-Ouest de la Côte d'Ivoire) : Essai de Correlation et Paleoenvironnements
}

\author{
J. W. D. Douzo, \\ K. T. S. Amalaman, \\ W. B. Dibo,
}

Département des Géosciences Marines, UFR des Sciences de la Terre et des Ressources Minières, Université Félix Houphouët Boigny, Côte d'Ivoire

Laboratoire des Géosciences et Techniques de l'Environnement, Département de Géologie, Faculté des Sciences, Université Chouaïb

Doukkali, Morocco
N. J.P. Yao,
K. L. Djeya,
C. L. Koffi,
Z. B. Digbehi,

Département des Géosciences Marines, UFR des Sciences de la Terre et des Ressources Minières, Université Félix Houphouët Boigny, Côte d'Ivoire

\section{A. Toufiq,}

Laboratoire des Géosciences et Techniques de l'Environnement, Département de Géologie, Faculté des Sciences, Université Chouaïb Doukkali, Morocco

R. Bié Goha,

Laboratoire des Sciences de la Terre et de l'Environnement, UFR de de

l'Environnement, Université Jean Lorougnon Guédé, Côte d'Ivoire

Doi: 10.19044/esj.2019.v15n15p401 URL:http://dx.doi.org/10.19044/esj.2019.v15n15p401

\section{Résumé}

Le matériel étudié dans ce travail est issu des déblais de cinq sondages hydrauliques implantés dans les localités de Fresco et de Grand-Lahou situées au Sud-Ouest dans la partie « onshore » du bassin sédimentaire ivoirien. Ces sondages s'inscrivent dans le cadre du programme national d'alimentation en eau potable à partir des nappes aquifères. Les analyses lithologiques et sédimentologiques des déblais de ces sondages sont réalisées en vue d'identifier les différentes unités lithologiques traversées, de comprendre les 
processus sédimentaires ayant prévalus lors du dépôt de ces sédiments et de mieux caractériser les paléoenvironnements de dépôt. L'étude lithologique a mis en évidence une sédimentation essentiellement argileuse et sableuse. La granulométrie des niveaux sableux montre qu'il s'agit des sables moyens à très grossiers, assez bien à mal classés, avec une asymétrie vers les éléments fins. Les courbes granulométriques indiquent une allure en forme sigmoïde « $\mathrm{S}$ » traduisant une origine fluviatile dans un environnement de dépôt à hydrodynamisme relativement moyen à fort. La morphoscopie des grains de quartz indique une dominance des grains émoussés luisants par rapport aux rond-mats et non usés, indiquant un transport dans un milieu aqueux loin de la source pourvoyeuse. A Grand-Lahou, la forte concentration des échantillons en carbonates suppose une mise en place dans un milieu marin peu profond et une sédimentation plus fine dans des conditions calmes. Les sédiments de Fresco montrent une teneur en carbonate faible, indiquant un milieu turbulent et défavorable à la précipitation du calcaire.

Mots-clés : Lithostratigraphie, sédimentologie, Fresco, Grand-Lahou, Paléoenvironnement 


\title{
Lithostratigraphical and Sedimentological Characterization of Deposits in the Fresco and Grand Lahou (Southwest Cote d'Ivoire): Correlation Attempt and Paleoenvironments
}

\author{
J. W. D. Douzo, \\ K. T. S. Amalaman, \\ W. B. Dibo,
}

Département des Géosciences Marines, UFR des Sciences de la Terre et des Ressources Minières, Université Félix Houphouët Boigny, Côte d'Ivoire

Laboratoire des Géosciences et Techniques de l'Environnement, Département de Géologie, Faculté des Sciences, Université Chouaïb

Doukkali, Morocco
N. J.P. Yao,
K. L. Djeya,
C. L. Koffi,
Z. B. Digbehi,

Département des Géosciences Marines, UFR des Sciences de la Terre et des Ressources Minières, Université Félix Houphouët Boigny, Côte d'Ivoire

\section{A. Toufiq,}

Laboratoire des Géosciences et Techniques de l'Environnement, Département de Géologie, Faculté des Sciences, Université Chouaïb Doukkali, Morocco

R. Bié Goha,

Laboratoire des Sciences de la Terre et de l'Environnement, UFR de de l'Environnement, Université Jean Lorougnon Guédé, Côte d'Ivoire

\section{Abstract}

The material studied in this work comes from excavations of five hydraulic soundings in the localities of Fresco and Grand-Lahou located in the Southwest in the "onshore" part of the Ivorian sedimentary basin. These surveys are part of the national program for drinking water supply from aquifers. The lithological and sedimentological analyzes of the cuttings of these holes are carried out in order to identify the different lithological units crossed, to understand the sedimentary processes that have prevailed during the deposition of these sediments and to better characterize the 
paleoenvironments of deposit. The lithological study revealed mainly clay and sandy in the sedimentation. The granulometry of the sandy levels shows that they are medium to a very coarse sand, fairly well classified, with asymmetry towards the fine elements. Granulometric curves indicate a sigmoidal "S" shape showing a fluvial origin in a relatively medium to strong hydrodynamic deposition environment. The morphoscopy of the quartz grains indicates a dominance of blunted grains that are shiny compared to the rounded and unworn grains, indicating a transport in an aqueous medium far from the source. At Grand-Lahou, the high concentration of carbonate samples implies emplacement in a shallow marine environment and finer sedimentation under calm conditions. The Fresco sediments show a low carbonate content, indicating turbulent and unfavorable medium for limestone precipitation.

Keywords: Lithostratigraphy, sedimentology, Fresco, Grand-Lahou, Paleoenvironment

\section{Introduction}

Depuis quelques décennies, la Côte d'Ivoire a intensifié la recherche pétrolière dans le bassin sédimentaire côtier afin de diversifier ses exportations en entrant résolument dans l'économie pétrolière. Les premiers suintements de bitumes dans la région d'Eboinda en 1950 ont marqué le début de l'exploration du pétrole en Côte d'Ivoire. La plupart des travaux réalisés par des géologues universitaires, en étroite collaboration avec les structures pétrolières, sur le bassin (Spengler et Delteil 1966) ne concernent que les dépôts du Crétacé du bassin offshore pour son intérêt pétrolier. Ces études ont permis de mettre en évidence le potentiel pétrolifère de la Côte d'Ivoire. D'autres relativement plus récentes (Digbehi et al., 1997 ; Saint-Marc et N'Da, 1997) ont à partir des données micropaléontologiques établi une synthèse biostratigraphique du bassin et ont apporté des précisions sur les environnements de dépôt. La biostratigraphie et les paléoenvironnements de l'intervalle Albien-Turonien et du passage Cénomanien-Turonien ont été étudiés à partir de sondages de la marge d'Abidjan (Bamba et al., 2011 ; Kouassi et al., 2013). Toutes ces études ont été menées dans la partie offshore du bassin ivoirien. Quelques études qui ont été réalisés sur la partie onshore du bassin (Bacchiana, 1982 ; Yao, 2012 ; Assalé, 2013 ; Kra, 2016) ont permis d'affiner l'échelle stratigraphique du bassin et les environnements de dépôts.

Ces travaux ont certes permis d'avoir des données importantes qui ont apporté des précisions stratigraphiques et des compléments d'analyses des paléoenvironnements sur les formations du bassin onshore au Sud de la faille des lagunes. Cependant les formations au Nord de cet accident majeur qui ne 
présentent pas d'intérêt pétrolier restent peu étudiées, d'où l'intérêt de de la présente étude.

L'objectif de ce travail est donc de définir le contexte paléoenvironnemental des dépôts de chaque unité lithologique en s'appuyant sur une étude stratigraphique et sédimentologique afin de reconstituer la dynamique des dépôts.

\section{Zone d'étude}

\subsection{Cadre géologique}

La région étudiée (Figure 1), appartient au bassin sédimentaire ivoirien qui représente $2,5 \%$ du territoire et couvrant une superficie de $30000 \mathrm{~km}^{2}$. Ce bassin s'étend de Sassandra (à l'Ouest) jusqu'à la frontière du Ghana (à l'Est). Cette partie (onshore) du bassin est très étroite à l'Ouest mais atteint $35 \mathrm{~km}$ de large au niveau d'Abidjan. Ce bassin est traversé d'Ouest en Est par une faille majeur, appelée « faille des lagunes » qui le divise en deux entités : - Au Nord, de cette faille les formations sont moins épaisses et formées des dépôts d'âge du «continental terminal » constitués d'argiles, de sables argileux et de grès plus ou moins ferrugineux (Digbehi 1987).

- Au Sud, les formations qui affleurent sont plus épaisses et sont recouvertes par des dépôts quaternaires (Affian 1986) composés de sables blancs et sable argileux continentaux, cordons sableux, de sables, d'argilites fluviolagunaires et des sables marins.

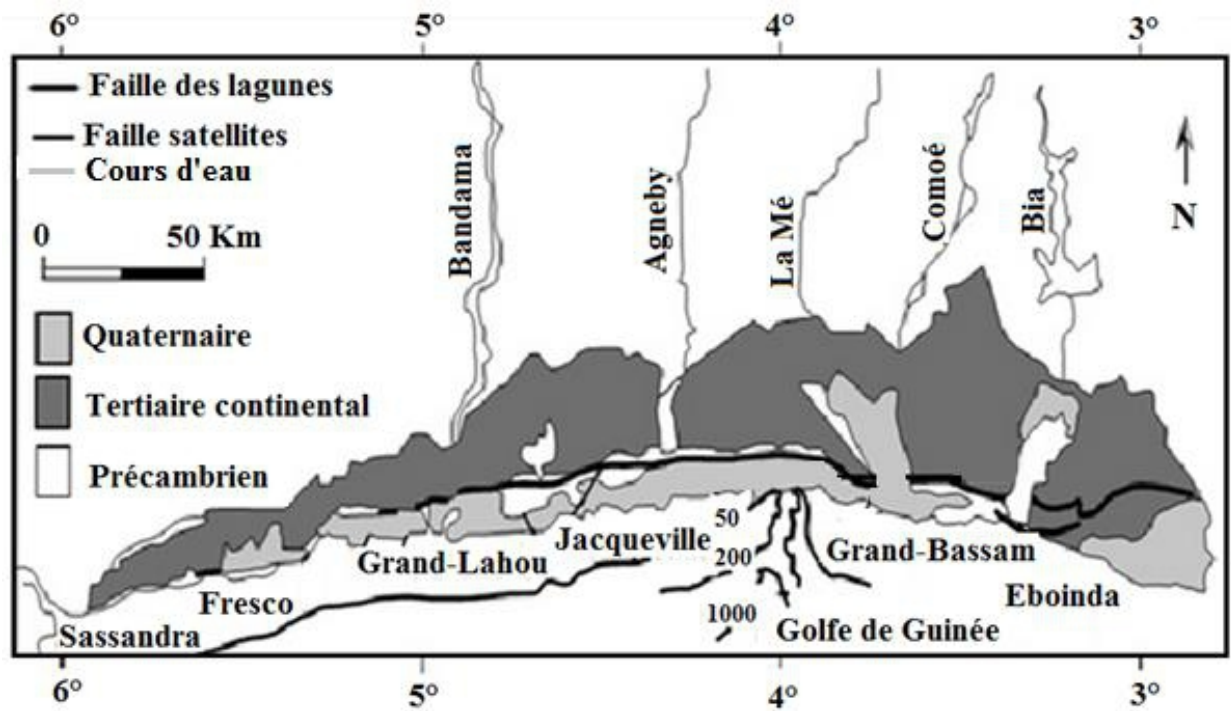

Figure 1 : Carte du Bassin sédimentaire onshore ivoirien (Hinschberger et Pomel 1979)

\subsection{Localisation et description des sondages}

Les sondages étudiés se situent dans la partie onshore du bassin sédimentaire ivoirien dans les localités de Grand-Lahou et de Fresco situées 
respectivement à $140 \mathrm{~km}$ et $200 \mathrm{~km}$ d'Abidjan. Les formations de Fresco et de Grand-Lahou sont d'âge crétacé inférieur à quaternaire (Soro 2011), cependant seules les formations du Tertiaire et du Quaternaire affleurent. A l'affleurement se rencontrent des dépôts du Continental Terminal (argiles, sables argileux, grès plus au moins ferrugineux), des dépôts quaternaires (sables grossiers des cordons littoraux), des sables et vases des fonds des lagunes et des faciès continentaux argilo-rubanés de la terre de barre ((Tastet, 1979 ; Stril, 1987 ; Aka 1991).

Les échantillons étudiés dans ce travail proviennent de deux sondages de Grand-Lahou (GL1 et GL2) et de trois sondages de Fresco (Fr1, Fr2, Fr3) dont les coordonnées géographiques sont indiquées dans le tableau 1. Les profondeurs des sondages varient de $25 \mathrm{~m}$ à $96 \mathrm{~m}$. Les prélèvements ont été réalisés sur place au cours des campagnes de forages. La figure 2 représente la localisation de ces différents sondages.

Tableau 1 : Position des sites d'implantation des sondages étudiés

\begin{tabular}{|l|l|l|}
\hline Sondages & Latitude (N) & Longitude (W) \\
\hline Fresco 1 (Fr1) & $05^{\circ} 06^{\prime} 21.6^{\prime}$ & $05^{\circ} 33^{\prime} 43.9^{\prime}{ }^{\prime}$ \\
\hline Fresco 2 (Fr2) & $05^{\circ} 06^{\prime} 10.7^{\prime}$, & $05^{\circ} 34^{\prime} 17.1^{\prime}$ \\
\hline Fresco 3 (Fr3) & $05^{\circ} 07^{\prime} 00^{\prime}{ }^{\prime}$ & $05^{\circ} 34^{\prime} 32,8^{\prime}$, \\
\hline Grand-Lahou 1 (GL1) & $05^{\circ} 14^{\prime} 44.7^{\prime}$ & $05^{\circ} 00^{\prime} 12.5^{\prime}$ \\
\hline Grand-Lahou 2 (GL2) & $05^{\circ} 14^{\prime} 47.1^{\prime}{ }^{\prime}$ & $05^{\circ} 02^{\prime} 39^{\prime}$ \\
\hline
\end{tabular}
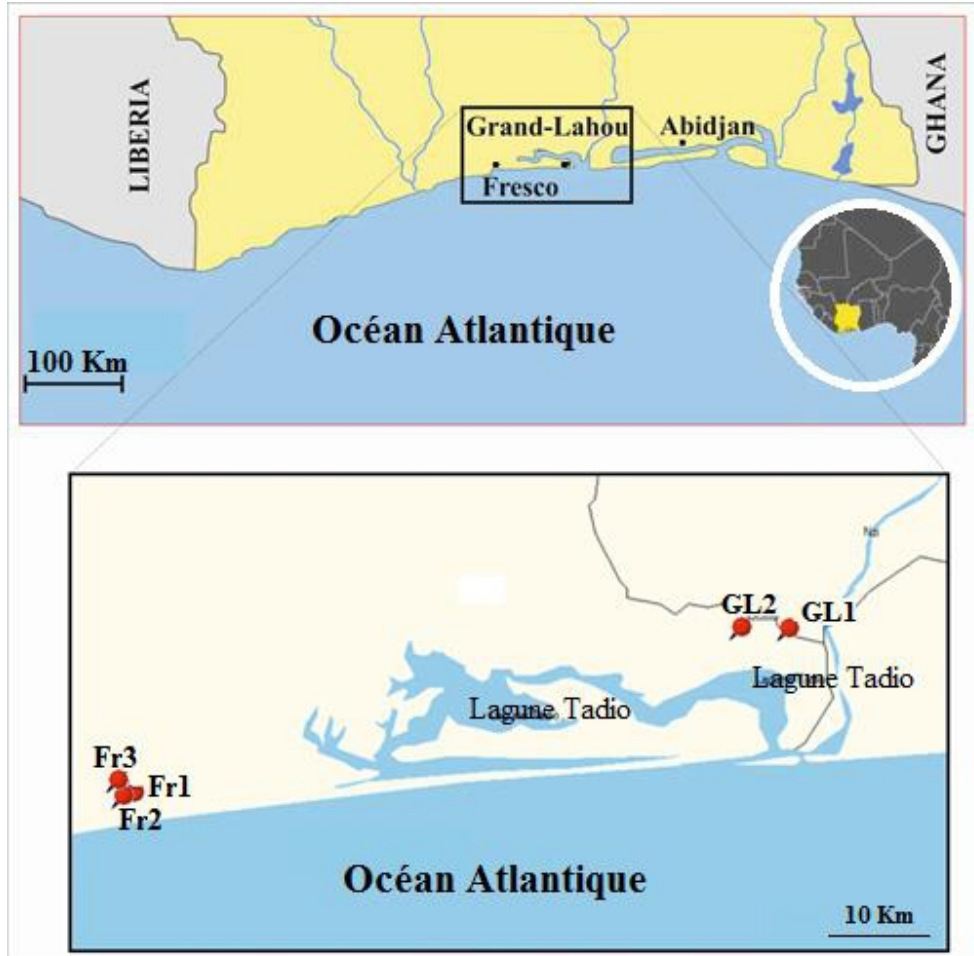

$\underline{\text { Légende }}$

- Villes

- Sondages

Cours d'eau

Figure 2 : Localisation des sondages de Fresco et de Grand-Lahou 


\section{Matériel et méthodes}

Le matériel utilisé est constitué de 281 échantillons de déblais de cinq (5) sondages implantés dans les localités de Fresco et Grand-Lahou. Ces échantillons ont fait l'objet d'une étude lithologique, granulométrique des fractions sableuses, morphoscopique des grains de quartz et calcimétrique pour doser les carbonates. L'analyse granulométrique des échantillons sableux a permis de déterminer la distribution granulométrique basée sur des techniques classiques (Vatan ,1967 ; Rivière, 1977 ; Chamley, 1988). Après lavage de chaque échantillon, les fractions sableuses sont séparées et traitées à $\mathrm{HCl}$ et $\mathrm{H}_{2} \mathrm{O}_{2}$ pour éliminer les carbonates et la matière organique, puis séchées dans une étuve à $60^{\circ} \mathrm{C}$. Après séchage, chaque échantillon de sable est tamisé sur une colonne de douze tamis vibrants (AFNOR) de maille variant entre $0,063 \mathrm{~mm}$ et $2 \mathrm{~mm}$. Le refus de chaque tamis est pesé puis les paramètres granulométriques (qui renseignent sur le degré de classement du sédiment : moyenne, écart type, Skewness) sont calculés (Cailleux et Tricart, 1959; Vatan, 1967 ; Rivière 1977). Les courbes granulométriques de fréquence et les courbes cumulatives sont également tracées. L'analyse morphoscopique (Cailleux et Tricart, 1959 ; Petit-John, 1957), par observation au microscope de type MOTIC SMZ-18, consiste en un examen de la forme générale des grains et de leurs degré d'usure, qui donneront des informations sur le transport et le milieu de dépôt. Le taux de carbonates dans les échantillons, mesuré à l'aide d'un calcimétre Bernard, permettra la mise en évidence des niveaux carbonatés.

\section{Résultats et Interprétations}

\subsection{Description lithologique}

Dans les zones de Fresco et de Grand-Lahou, les dépôts sont dominés par les faciès sableux et argileux. L'observation macroscopique des sédiments a permis de mettre en évidence quatre faciès sédimentaires (Figure 3) : les sédiments mixtes (argiles sableuses et sables argileux), les argiles, les grès et les sables; le tout recouvert par une couche de terre végétale brune peu évoluée.

Les principales composantes lithologiques observées dans les différents sondages sont les argiles, les sables (et grès) et les faciès mixtes.

\section{- $\quad$ Argiles}

Présentes dans tous les sondages, elles sont particulièrement représentées dans les sondages de Grand-Lahou, avec des épaisseurs allant de $2 \mathrm{~m}$ à $42 \mathrm{~m}$, elles sont de couleur rouge, noire, grise, brune ou verte. La couleur rouge pourrait s'expliquer par la richesse en oxyde de Fer. La couleur noire est due en partie à l'apport de la matière organique produite par la dégradation des débris végétaux. Quant à la coloration verte de l'argile à la base de la 
sédimentation de Grand-Lahou, elle est due à l'abondance de la glauconite. Par endroit, les argiles sombres sont intercalées de calcaires. Les argiles grises et verdâtres sont uniquement observées dans les sondages de Grand-Lahou. Ces argiles sont relativement compactes riches en pyrite, glauconite, débris carbonés et paillettes de micas. Des débris de coquilles de bivalves, et de gastéropodes, des ostracodes, des foraminifères benthiques et de quelques petits foraminifères planctoniques y sont également observés.

\section{- $\quad$ Sables}

Ils constituent des niveaux en nombre assez important dans tous les sondages étudiés. Les sables quartzeux dans ces sondages sont en majorité blancs, avec une faible présence de grains de quartz d'autre couleur (rouge, gris-clair, jaune-orangé et ocre). Leurs épaisseurs varient de $2 \mathrm{~m}$ (sondage $\mathrm{Fr} 2$ ) à $15 \mathrm{~m}$ (sondage GL1). Les grains de quartz sont en majorité sub-arrondis, arrondis à subanguleux, les anguleux sont rares. Ils sont pour la plupart émoussés luisants, leur taille va du très grossiers à fins. Des cristaux de pyrite, de la glauconite, des paillettes de micas et des débris carbonés se rencontrent dans la quasi-totalité des niveaux sableux où l'on note également la présence de coquilles brisées.

\section{Grès}

Observés uniquement au sondage de Grand-Lahou 1, d'une épaisseur de $5 \mathrm{~m}$ entre les profondeurs $69 \mathrm{~m}$ et $74 \mathrm{~m}$, ils constituent un banc consolidé de couleur gris-clair à ciment marneux. Les grains de quartz sont enfumés, très grossiers à moyens. La majorité des formes sont sub-anguleux à anguleux. On note aussi la présence de glauconite, de pyrite, des débris carbonés, et coquilliers. Les proportions de foraminifères benthiques et d'ostracodes bien conservés sont faibles. La matrice argileuse est de couleur brune, sa teneur en carbonates varie de 5 à 21,67\% dans cet intervalle.

\section{- $\quad$ Faciès mixtes (argile sableuse et sable-argileux)}

Les faciès mixtes (sables argileux et argiles sableuses) représentent plus de $60 \%$ de la sédimentation. Les argiles sableuses sont souvent rougeâtres et donc très oxydées. Les grains de quartz y sont majoritairement grossiers à fins, subanguleux à arrondis, translucides, luisants.

Les sables argileux sont de couleur variable (blanchâtre, jaunes-orangé à rougeâtre), ils contiennent des grains de quartz arrondis à subarrondis ou subanguleux, les anguleux sont rares. La fraction argileuse est rouge, jauneorangé ou ocre, très altérée et friable, pauvre en carbonates. La pyrite, les débris carbonés, les débris de coquilles et les paillettes de micas y sont fréquents. 


\section{Synthèse lithologique des formations}

Sur la base des données obtenues de l'observation macroscopique, de l'observation à la loupe binoculaire des déblais lavés et séchés puis des données de calcimétrie, les colonnes lithologiques ont été subdivisées en cinq unités. On note l'absence de certaines unités sédimentaires dans les sondages de Grand-Lahou et de Fresco.

\section{- Unité 1: Argiles sombres à passées calcaires, grès et sable argileux}

La base de cette unité montre des argiles sombres avec passées calcaires parfois riches en glauconite. Ces argiles sont très compactes et bien exprimée dans le sondage de Grand-Lahou 2 (42 m), où elle se termine par un niveau d'argile grise friable. A Grand-Lahou 1 ces argiles sont intercalées par un banc de grès assez grossiers à ciment argileux $(5 \mathrm{~m})$ et sont surmontées par deux niveaux de sable argileux séparés par un niveau d'argile sombre et de sableux.

\section{Unité 2: Argile sombre}

Cette unité d'argiles est plus exprimée dans les sondages de Fresco. Elle apparait nettement dans le sondage Fresco 1 (10 m d'épaisseur), partiellement dans le sondage Fresco $3(7 \mathrm{~m})$; à GL1 sont épaisseur ne dépasse pas 2 mètres, elle vraisemblablement érodée dans le sondage Grand-Lahou 2.

\section{Unité 3: Sable argileux, sable et argile sableuse}

Cette unité est plutôt représentée dans les sondages de Fresco, elle comporte une succession de trois faciès dont le sable argileux, le sable et l'argile sableuse. La base de cette unité est constituée de sable argileux et d'argile sableuse dans les sondages de Fresco 1 et de Fresco 3, d'argile sableuse dans le sondage de Grand-Lahou 1. Ces niveaux sont surmontés d'un banc de sable suivi de sables argileux. Cette unité n'est pas atteinte dans le sondage de Fresco 2, elle est absente dans le sondage de Grand-Lahou 2.

\section{- $\quad$ Unité 4: Cuirasse latéritique}

Cette unité constitue la partie sommitale des sondages. Sa base comporte des bancs de sable surmontés de niveaux sablo-argileux et argilosableux. A son sommet s'observent des argiles latéritiques avec présence de cuirasses ferrugineuse. Cette unité s'observe dans tous les sondages elle est particulièrement épaisse dans le sondage Grand-Lahou 1 (7 m).

\section{- Unité 5: Terre végétale}

Cette unité, aussi appelée « Terre de barre », coiffe tous les sondages. Elle est constituée d'argile très oxydée, de sable et de silts. Elle est très silteuse et sa couleur varie du jaune orangé à l'orange jaunâtre pâle. Pour les 
formations les plus superficielles, la coloration devient rouge brunâtre. Elles contiennent des cuirasses ferrugineuses, des débris carbonés, des morceaux de tige et des racines. Les grains de quartz sont rouge, translucides, anguleux à subanguleux.

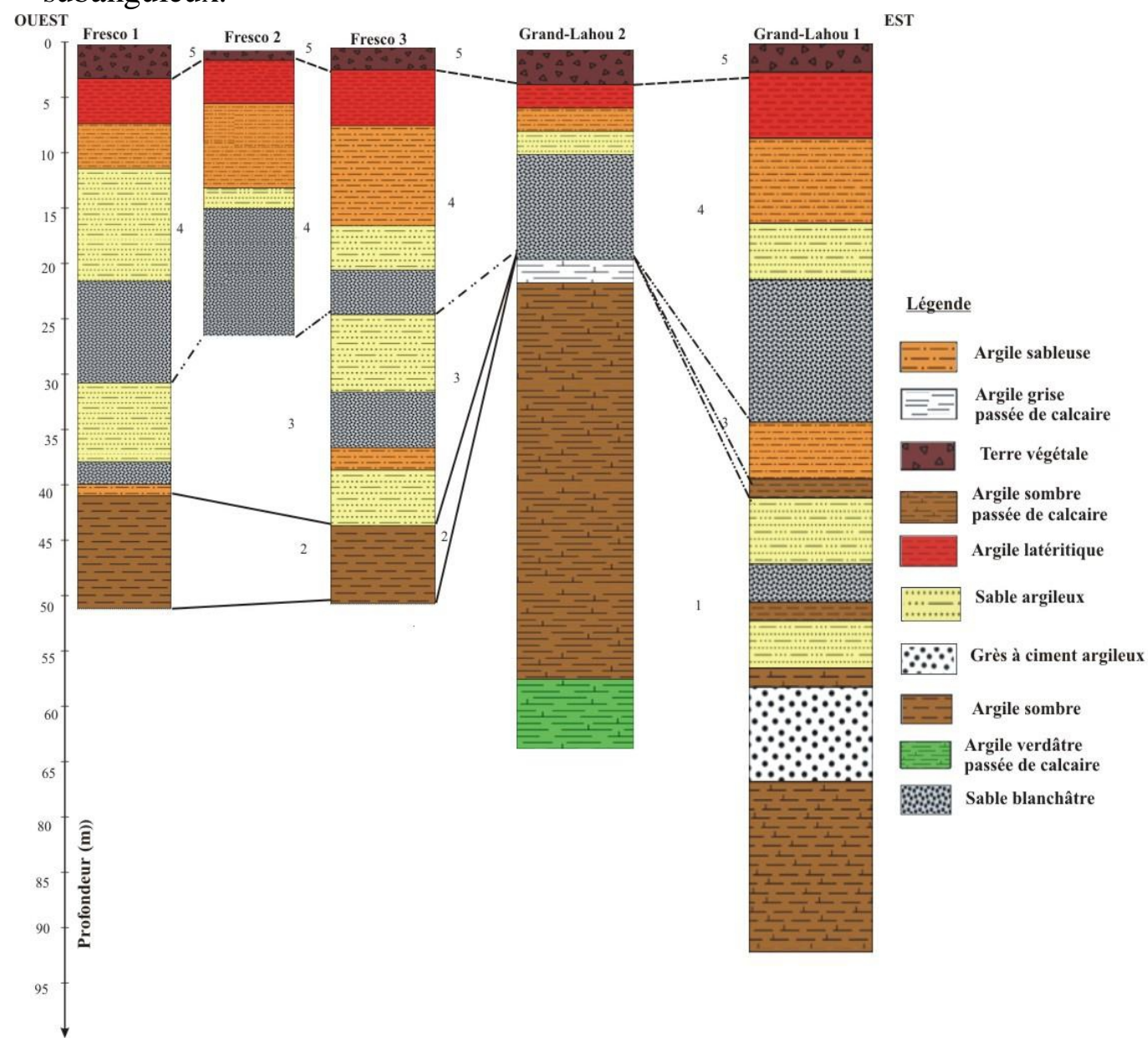

Figure 3 : Essai de corrélation lithologique des sondages de Fresco et de Grand-Lahou

\subsection{Etude granulométrique}

\subsubsection{Paramètres granulométriques}

Les valeurs représentées dans le tableau 2 indiquent les paramètres granulométriques de quelques échantillons des sondages de Fresco et de Grand-Lahou. Les données relatives à la taille des grains, ainsi que les paramètres caractéristiques (moyenne, écart-type, Skewness) des échantillons de sable collectés, sont représentées dans les figures 4 à 6 .

- $\quad$ La moyenne de la taille des grains varie entre $514 \mu \mathrm{m}$ et $1114 \mu \mathrm{m}$, elle indique des sables très grossiers à moyens. 
- $\quad$ L'écart-type évoque des valeurs inférieures ou proches de 1, traduisant des sables assez bien classés à médiocrement classés en passant par les sables moyennement classés (figure 5).

Les valeurs du Skewness sont généralement négatives dans les sondages de Fresco 2 et Grand-Lahou 2 ( $-1,2$ et 0$)$, positives dans les sondages de Fresco 1 et Grand-Lahou 1 (0 et 2,5) et dans le sondage de Fresco 3 les valeurs oscillent entre $(-0,2$ et 0,4$)$ (figure 6) Ces valeurs montrent que l'étalement se fait du côté de la fraction fine.

Tableau 2 : Paramètres granulométriques des dépôts des sondages de Fresco et de Grand-

Lahou

\begin{tabular}{|c|c|c|c|c|c|c|}
\hline Localités & Sondages & $\begin{array}{l}\text { Profondeur } \\
\text { (m) }\end{array}$ & Moyenne & $\begin{array}{l}\text { Ecart-type } \\
(\sigma)\end{array}$ & Skewness(Sk) & Description / Interprétation \\
\hline \multirow{6}{*}{ 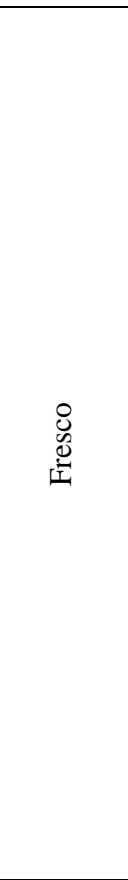 } & \multirow[t]{2}{*}{ Fr1 } & $11 ; 20$ & $933 ; 566$ & $1.13 ; 0.53$ & $-0.2 ; 1.25$ & $\begin{array}{l}\text { Sables grossiers, assez bien } \\
\text { classés à médiocrement classés, } \\
\text { très asymétriques vers les } \\
\text { éléments fins et asymétriques } \\
\text { vers les éléments fins. }\end{array}$ \\
\hline & & $21 ; 30$ & $632 ; 659$ & $0.44 ; 0.57$ & $1.11 ; 2.02$ & $\begin{array}{c}\text { Sables grossiers, très bien } \\
\text { classés à assez bien classés, très } \\
\text { asymétriques vers les éléments } \\
\text { fins. }\end{array}$ \\
\hline & \multirow[t]{2}{*}{ Fr2 } & $11 ; 14$ & $515 ; 433$ & $0.92 ; 0.65$ & $-0.36 ;-0.44$ & $\begin{array}{l}\text { Sables grossiers à moyens, assez } \\
\text { bien classé à moyennement } \\
\text { classés, très asymétriques vers } \\
\text { les éléments grossiers. }\end{array}$ \\
\hline & & $15 ; 25$ & $413 ; 533$ & $0.62 ; 0.36$ & $-0.46 ; 0.2$ & $\begin{array}{c}\text { Sables grossiers à moyens, bien } \\
\text { classés et très asymétriques vers } \\
\text { les éléments grossiers et } \\
\text { asymétriques vers les éléments } \\
\text { fins. }\end{array}$ \\
\hline & \multirow[t]{2}{*}{ Fr3 } & $19 ; 29$ & $742 ; 579$ & $0.7 ; 0.8$ & $0.14 ; 0.2$ & $\begin{array}{c}\text { Sables grossiers, bien à assez } \\
\text { bien classés, très asymétriques } \\
\text { vers les éléments fins. }\end{array}$ \\
\hline & & $34 ; 40$ & $514 ; 557$ & $1.4 ; 0.7$ & $-0.1 ; 0.2$ & $\begin{array}{l}\text { Sables moyens, médiocrement } \\
\text { classés, asymétriques vers les } \\
\text { éléments fins }\end{array}$ \\
\hline \multirow{4}{*}{ 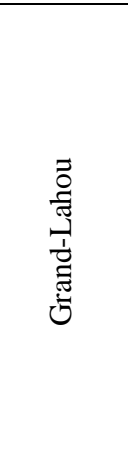 } & \multirow[t]{2}{*}{ GL1 } & $20 ; 25$ & $1094 ; 1331$ & $0.79 ; 0.57$ & $0.2 ; 0.38$ & $\begin{array}{l}\text { Sables grossiers, assez bien } \\
\text { classés asymétriques vers les } \\
\text { éléments fins }\end{array}$ \\
\hline & & $27 ; 40$ & $1104 ; 1114$ & $0.96 ; 1.08$ & $0.4 ; 0.5$ & $\begin{array}{l}\text { Sables très grossiers, assez bien } \\
\text { classés, asymétriques vers les } \\
\text { éléments fins. }\end{array}$ \\
\hline & \multirow[t]{2}{*}{ GL2 } & $7 ; 9$ & $682 ; 810$ & $1.10 ; 1.08$ & $-0.2 ;-0.1$ & $\begin{array}{l}\text { Sables grossiers médiocrement } \\
\text { classés, presque asymétriques } \\
\text { vers les éléments grossiers. }\end{array}$ \\
\hline & & $10 ; 18$ & $834 ; 1006$ & $1.09 ; 1.2$ & $-0.23 ;-0.1$ & $\begin{array}{l}\text { Sables très grossiers à grossiers, } \\
\text { médiocrement classés, } \\
\text { asymétriques vers les éléments } \\
\text { grossiers. }\end{array}$ \\
\hline
\end{tabular}



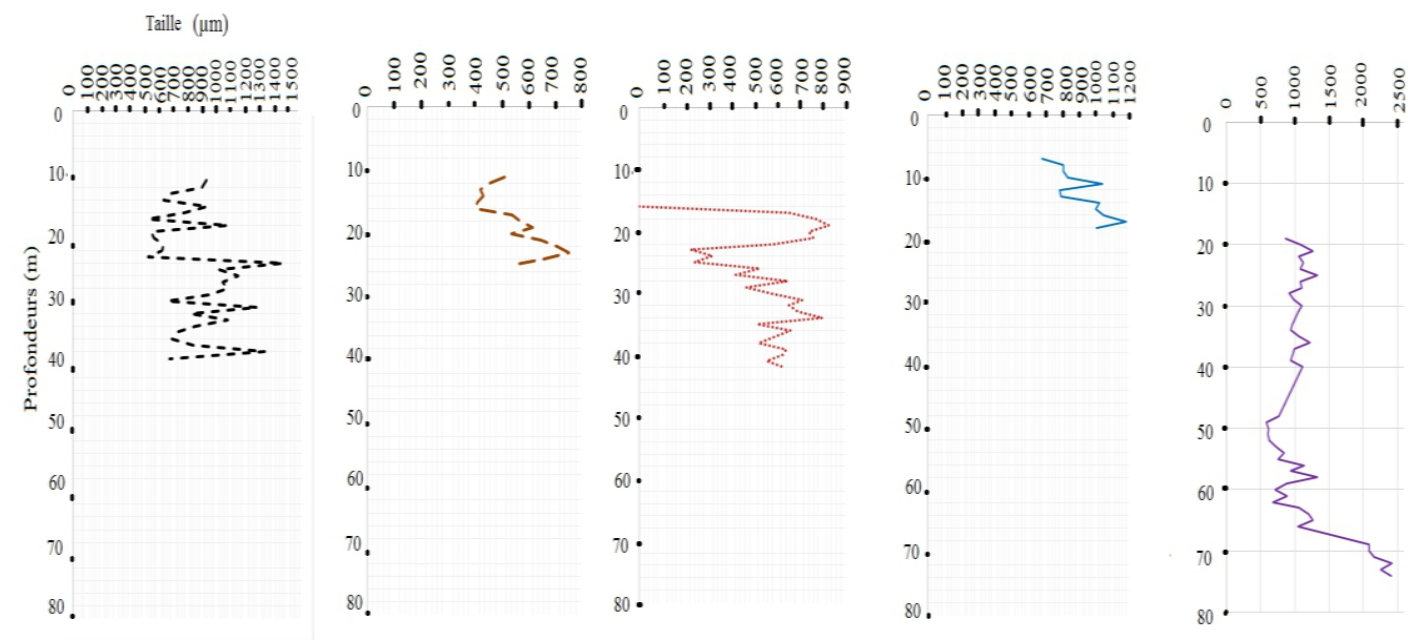

Fresco 1

Fresco 2

Fresco 3

Grand-Lahou 2

Grand-Lahou 1

Figure 4: Taille moyenne des grains de quartz dans les sondages de Fresco et de GrandLahou

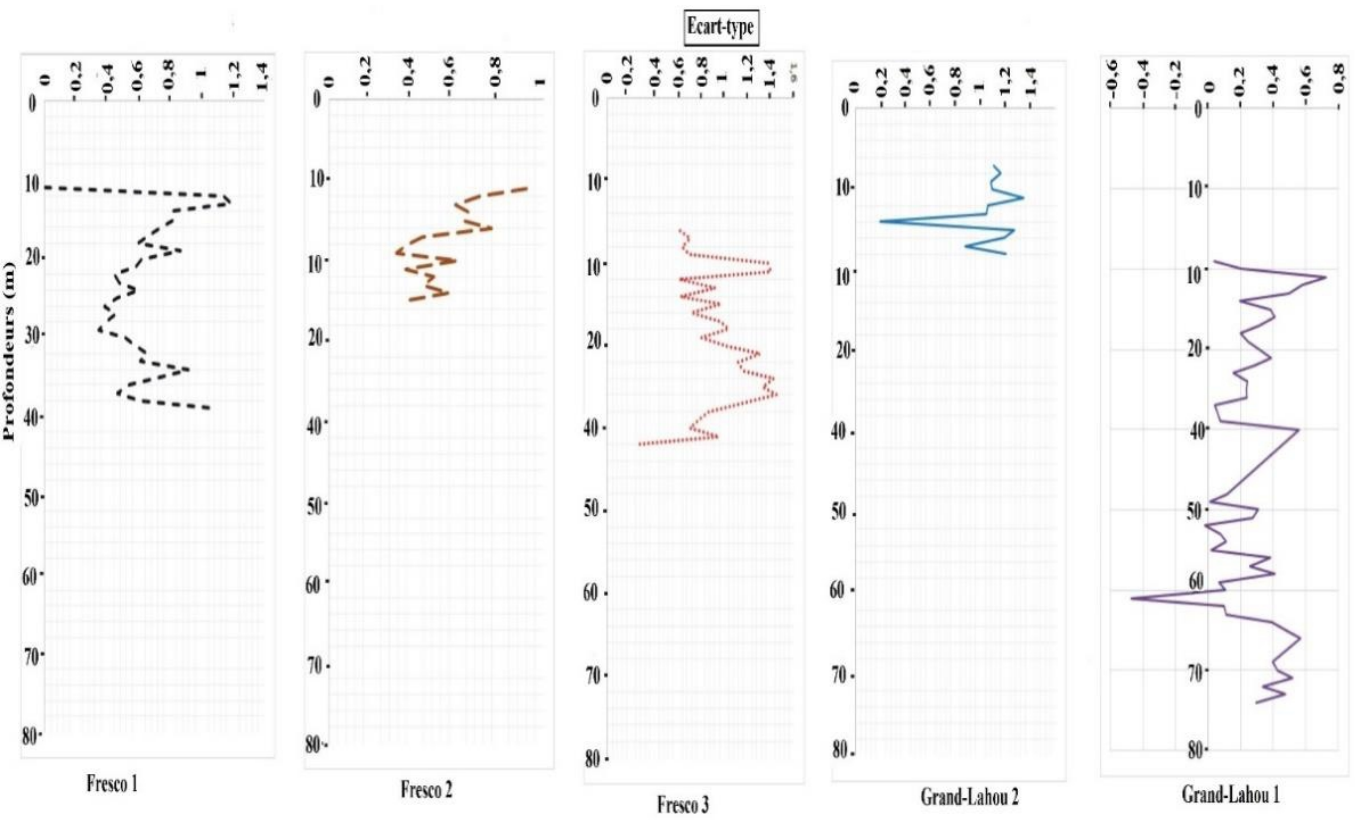

Figure 5 : Ecart type des dépôts de sable dans les sondages de Fresco et de Grand-Lahou 


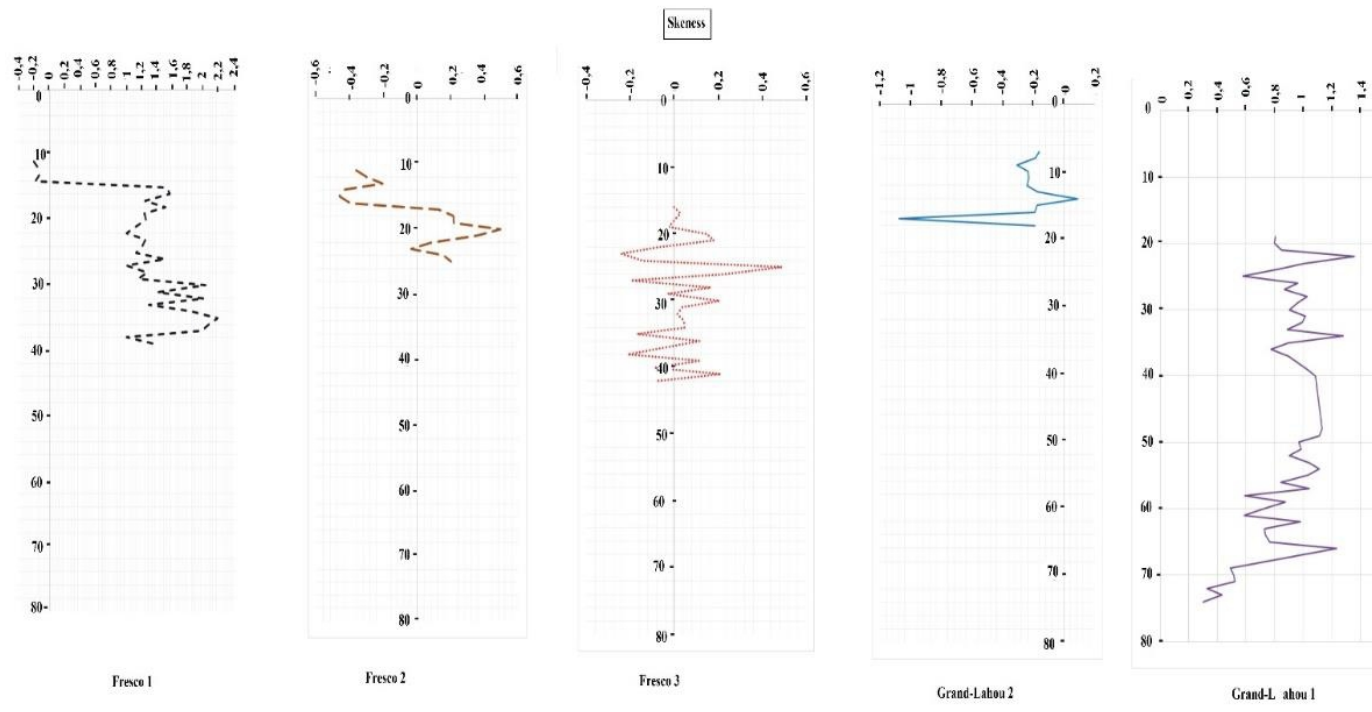

Figure 6: Skewness des dépôts de sable dans les différents sondages de Fresco et de Grand-Lahou

Les paramètres granulométriques montrent qu'à Fresco, les sables sont en majorité grossiers à rarement moyens. Ils sont assez bien classés et asymétriques vers les éléments fins en raison de l'afflux sédimentaire caractérisant un milieu à haute énergie et les apports préférentiels des sédiments fins.

A Grand-Lahou, les sables sont plutôt très grossiers à grossiers, prouvant une dynamique très forte. Ils sont assez-bien classés à médiocrement classés, preuve d'un régime hydrodynamique variable. Ils sont asymétriques vers les éléments fins et rarement vers les éléments grossiers. Les valeurs enregistrées traduisent dans l'ensemble un hydrodynamisme variable dominé par un régime moyen à faible.

\subsubsection{Faciès granulométriques}

Les courbes granulométriques cumulatives des fractions sableuses brutes des différents sondages (Figure 7) de Fresco et de Grand-Lahou sont identiques, elles présentent les mêmes allures de types sigmoïdes. Elles sont tournées vers le bas, ce type de courbes témoigne d'un environnement calme et une sédimentation par décantation (Defaflia et al., 2013). Selon Tricard (1965), ces courbes relativement peu redressées témoignent d'un faciès sigmoïde. Cela indique une sédimentation réalisée par accumulation libre, les grains gossiers et moyens étant transportés sur le fond de l'eau par roulement ou par saltation et les grains fins par suspension. Ce faciès révèle donc une réduction progressive de l'agent de transport exprimant un hydrodynamisme 
moyen à faible. Pour Pinot (1994), la forme « $\mathrm{S}$ » est dénommée faciès par excès de charge et serait un faciès de sable littoral où les grains sont transportés par saltation.
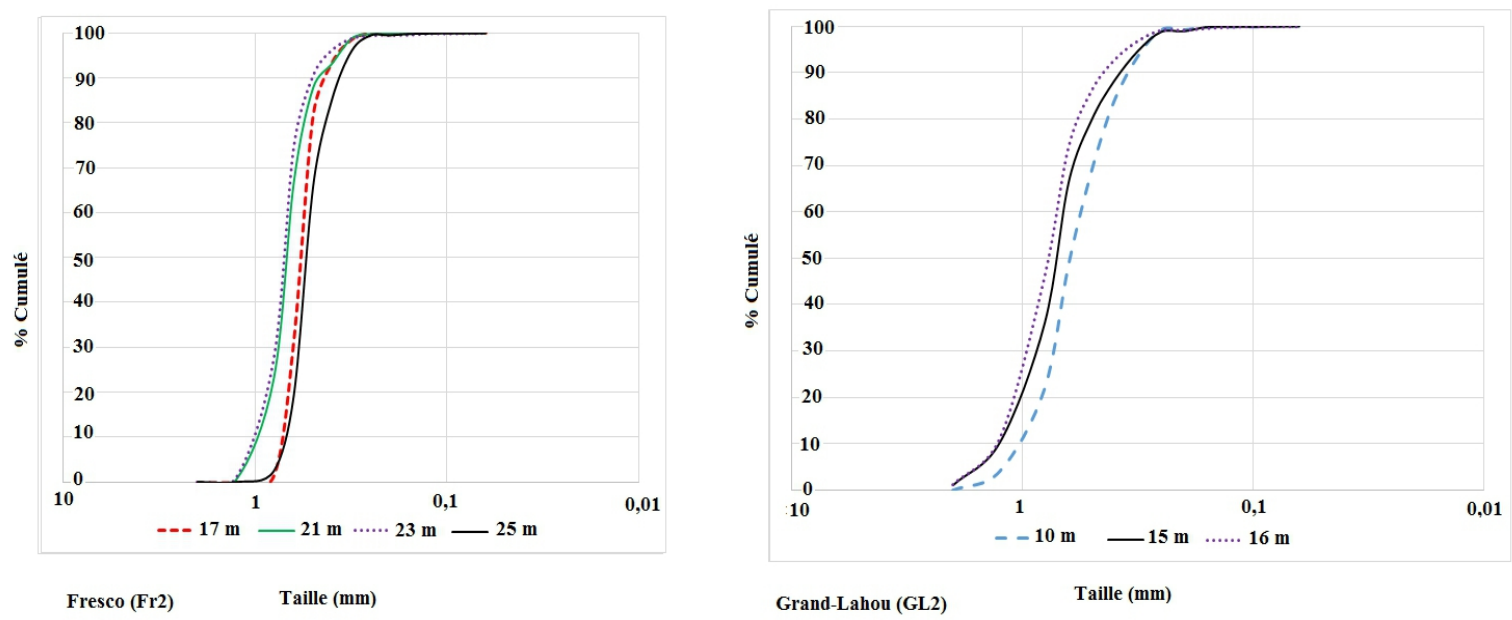

Figure 7 : Courbes granulométriques cumulatives des fractions sableuses des sondages de Fresco et de Grand-Lahou

\subsubsection{Analyse granulométriques des dépôts des sondages de Fresco et de Grand-Lahou}

Les courbes de fréquences granulométriques (Figure 8 et 9) des échantillons de Fresco et de Grand-Lahou analysés sont bimodales, trimodale ou unimodale. Ces résultats montrent que les sédiments sont hétérogènes. L'aspect polymodal des courbes traduit une intervention de plusieurs processus hydrodynamiques lors de la mise en place de ces niveaux sableux. Les grains de quartz sont polygéniques car ils sont d'origine variée. Plus le mélange est constitué de grains de tailles différentes, plus l'allure présente plusieurs modes traduisant un mélange de sédiments d'origines différentes. Les courbes monomodales (figure 8 et 9) sont caractéristiques des sédiments fins et suggèrent une homogénéité des sédiments. Les grains sont issus d'une seule source et par conséquent le milieu est plus ou moins fermé (Fresco 1, Grand-Lahou 2). 
Fresco 1 (25 m)

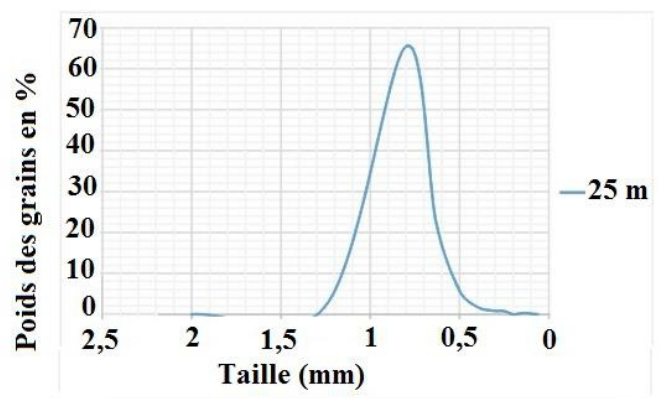

Grand-Lahou 1 (25 m)

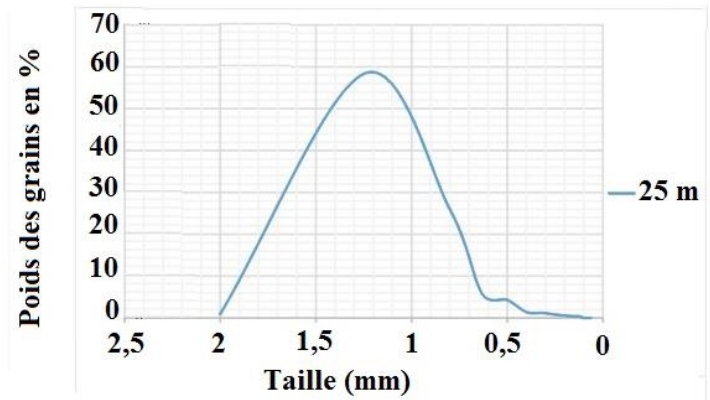

Fresco 2 (14 m)

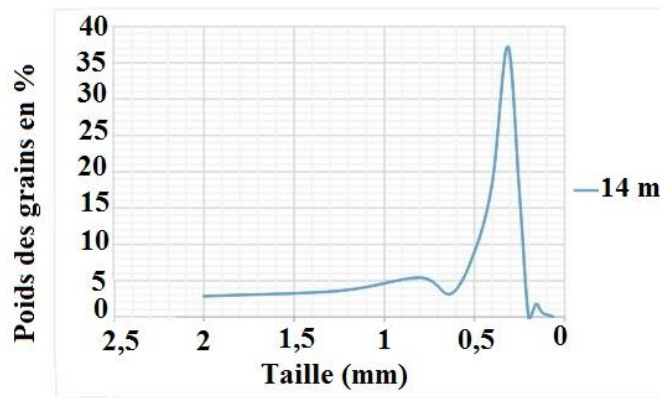

Grand-Lahou 2 (10 m)

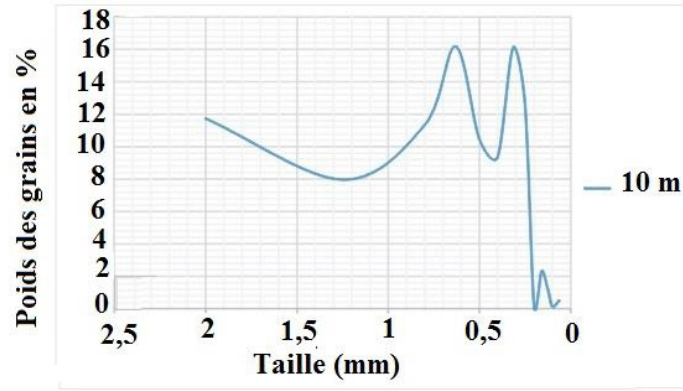

Figure 8: Courbe granulométriques simple de fréquences de dépôts des sondages de Fresco et de Grand-Lahou

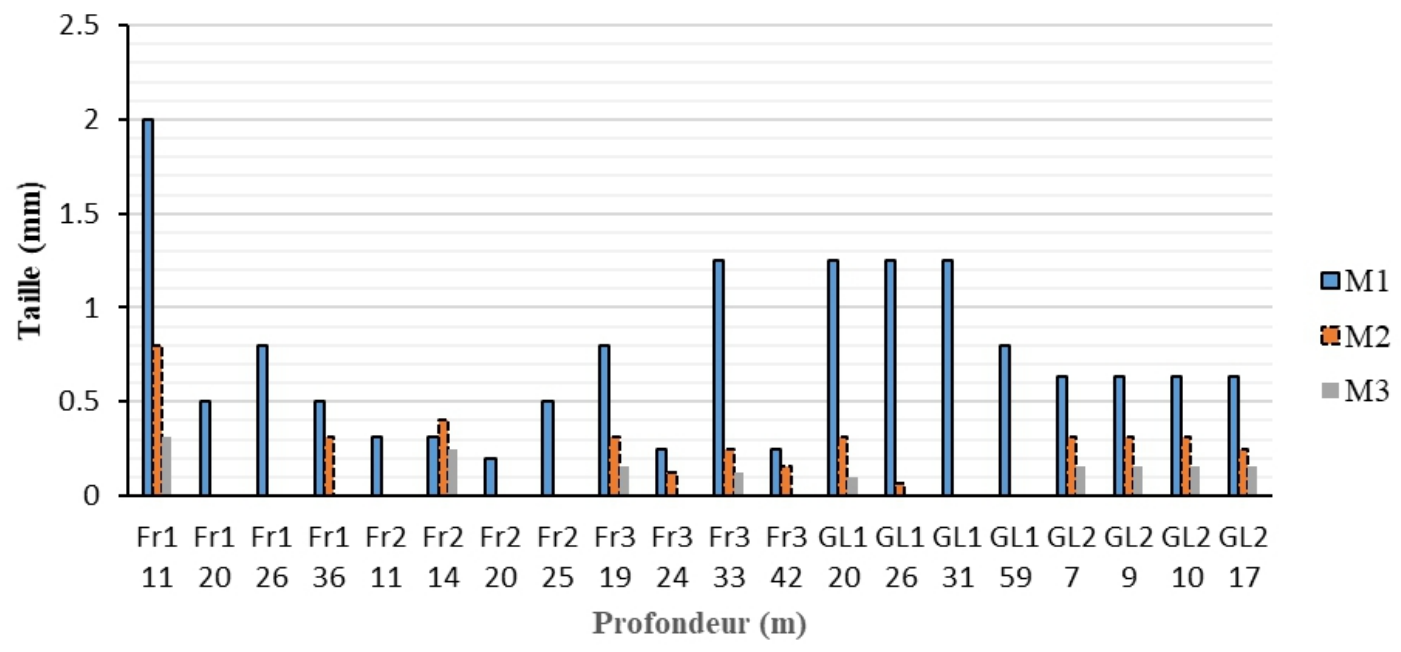

Figure 9: Evolution des modes de sable dans les sondages de Fresco et de Grand-Lahou

\subsection{Morphoscopie des grains de quartz}

L'étude morphoscopique des grains de quartz (Figure 10) dans les sondages de Fresco et de Grand-Lahou (Tableaux 4) montre une prédominance des grains émoussés luisants (EL) avec une moyenne de 67,7 
$\%$. Ce résultat montre l'influence du transport par l'eau et une dynamique fluviatile sur les grains de sables. Selon Cailleux et Tricard (1959), les grains émoussés luisants sont le résultat du transport sur une longue distance en milieu aquatique tel que révélé dans ce travail (transport fluviatile), le milieu peut correspondre à un littoral. Les grains ronds mats $(\mathrm{RM})$ représentent en moyenne $24 \%$ et les grains non usés (NU) représentent 8,84\%. Leur présence avec des sédiments peu évolués reflète une source proche mais aussi un important remaniement des sédiments avant leur dépôt final. Les grains ronds mats dans les dépôts indiquent un transport de ces derniers par le vent. La présence d'oxyde ferrique d'origine continentale donne cette couleur rouge, jaune orangé à roux aux grains de quartz.
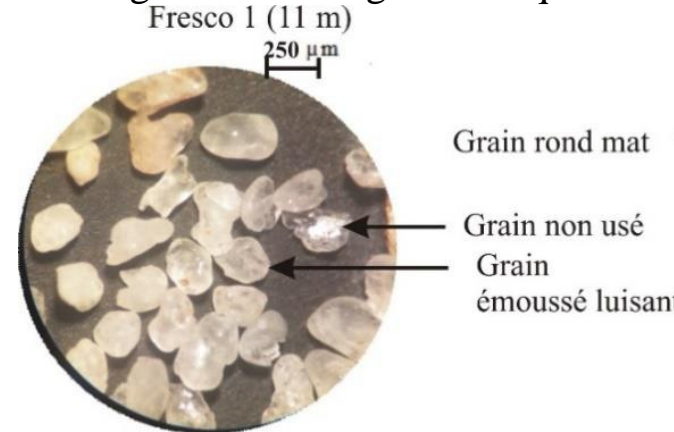

A: Fresco

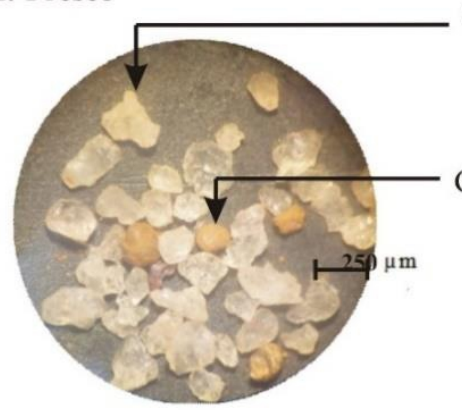

Grand-Lahou 1 (20 m)

B: Grand-Lahou

Grain non usé

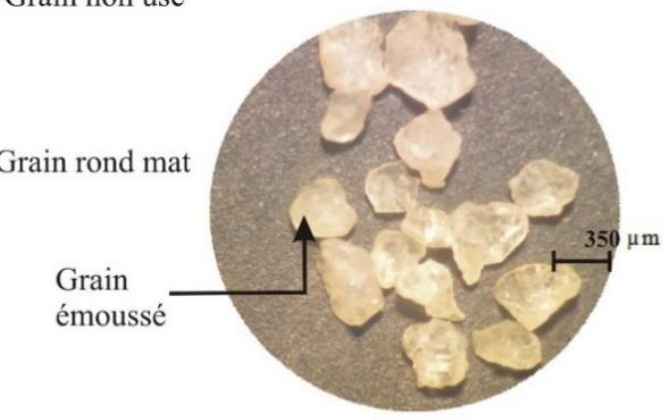

Grand-Lahou 2 (10 m)

Figure 10 : Morphologie des grains de quartz des dépôts des sondages de Fresco et de Grand-Lahou 
Tableau 4: Pourcentage des morphologies de grains de quartz des dépôts dans les sondages de Fresco et de Grand-Lahou

\begin{tabular}{|c|c|c|c|c|c|}
\hline Localité & Sondage & $\begin{array}{l}\text { Profondeur } \\
(\mathrm{m})\end{array}$ & $\begin{array}{l}\text { Non } \\
\text { usés } \\
(\%)\end{array}$ & $\begin{array}{l}\text { Rond } \\
\text { Mats } \\
((\%)\end{array}$ & $\begin{array}{l}\text { Emoussés } \\
(\%)\end{array}$ \\
\hline \multirow{6}{*}{ 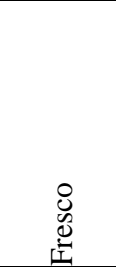 } & \multirow[b]{2}{*}{ Fr1 } & 11 & 12 & 19 & 69 \\
\hline & & 20 & 5 & 22 & 73 \\
\hline & \multirow[b]{2}{*}{$\mathrm{Fr} 2$} & 14 & 13 & 27 & 60 \\
\hline & & 25 & 6 & 31 & 63 \\
\hline & \multirow[b]{2}{*}{ Fr3 } & 19 & 9 & 11 & 83 \\
\hline & & 33 & 8 & 15 & 77 \\
\hline \multirow{4}{*}{ 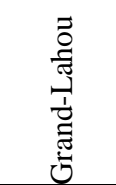 } & \multirow[b]{2}{*}{ GL1 } & 20 & 8 & 32 & 60 \\
\hline & & 31 & 15 & 30 & 55 \\
\hline & \multirow[b]{2}{*}{ GL2 } & 10 & 2 & 35 & 63 \\
\hline & & 17 & 6 & 20 & 74 \\
\hline \multicolumn{3}{|c|}{ Pourcentage moyen (\%) } & 8,84 & 24,2 & 67,7 \\
\hline
\end{tabular}

\subsection{Calcimétrie}

L'étude calcimétrique des sédiments met en évidence trois intervalles (Figure 11) dont deux carbonatés et un non carbonaté. Les niveaux carbonatés ne se rencontrent que dans les sondages de Grand-Lahou, avec des teneurs en $\mathrm{CaCO}_{3}$ variant de $5 \%$ à $40 \%$. Les sondages de Fresco sont pauvres en carbonates $(\leq 2,5 \%)$. Les niveaux superficiels, constitués d'argiles latéritiques et sableuses, de sables argileux et de sables grossiers ne renferment pas de carbonates, le milieu est probablement agité ou turbulent.

A Grand-Lahou, seuls les niveaux argileux et gréseux sont carbonatés (30 à $40 \%$ de $\left.\mathrm{CaCO}_{3}\right)$.

Les forts taux de carbonates enregistrés évoquent un milieu de sédimentation calme ou à faible énergie, favorisant la précipitation chimique des calcaires. La chute du taux de carbonates par endroit indique une augmentation sensible de l'énergie du milieu. 


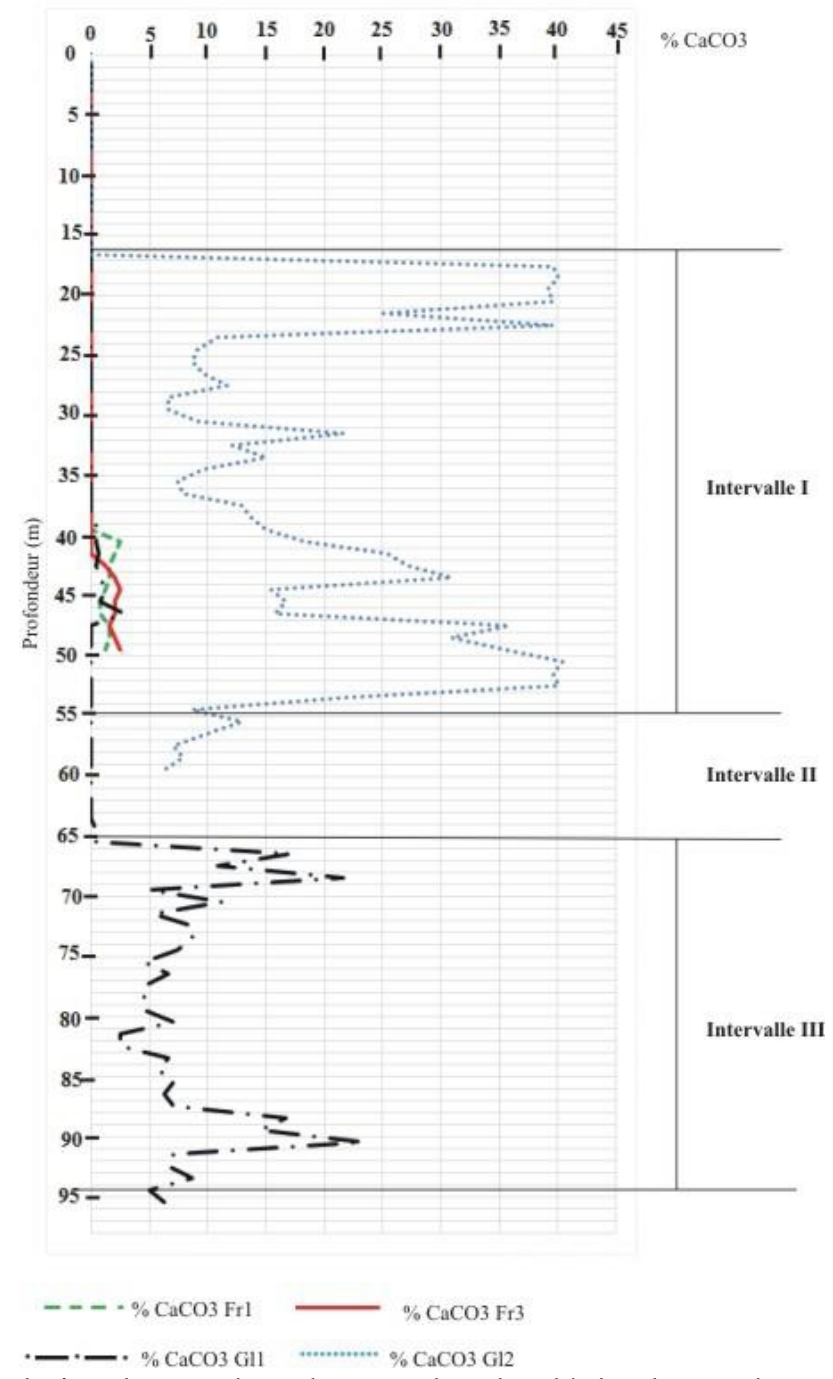

Figure 11: Evolution du taux de carbonates dans les dépôts des sondages de Fresco et de Grand-Lahou

\section{Discussions}

\section{- Lithologie}

Les études lithologiques des sondages de Fresco et de Grand-Lahou ont mis en évidence quatre faciès dont l'argile, le sable, les grès, et les sédiments mixtes (sable argileux et argile sableuse), le tout coiffé par l'argile latéritique et de terre végétale. Les dépôts de Grand-Lahou sont plus riches en argiles que ceux de Fresco (qui sont plus sableux), traduisant un milieu de dépôts agité à Fresco et plus calme à Grand-Lahou. Les sédiments à grains grossiers indiquent une énergie hydrodynamique élevée. Ils sont interprétés comme des dépôts résiduels de la base des chenaux fluviatiles (Flores et al., 1985; Miall, 1996), ou issus d'un milieu turbulent (Yao, 2012). La présence 
de sables, d'argiles et de grès dans les deux localités caractérise un environnement à énergie de dépôt variable. Les dépôts sableux intercalent les argiles dans la majorité des sondages (GL1, GL2, Fr1, Fr3). Selon Lowe (1982) et Cremer (1983), le dépôt de sables grossiers intercalés d'argile est dû aux variations de l'énergie de dépôt qui augmente (lorsque se manifeste un courant de haute turbulence) conduisant à des sédiments sableux ou qui diminue pour les argiles. Gbangbot (2012) stipule que lorsque le dépôt de sable repose sur la formation d'argile cela exprime un milieu à fort hydrodynamisme.

L'alternance de faciès argileux et sableux dans le sondage de GrandLahou 1 suggère ainsi un hydrodynamisme local très contrasté avec, soit une variation de l'intensité des crues, soit des déplacements latéraux des lits de rivières en accord avec Vigreux et al. (2011). Les brusques changements de l'énergie et de la compétence du courant d'écoulement provoquent l'alternance des niveaux sableux et argileux (Kra, 2016).

L'épaisse couche d'argile noire des sondages de Grand-Lahou est due au processus de décantation, témoignant d'un milieu de dépôt à régime hydrodynamique faible, voire nul (Yao, 2012). Les sédiments fins sont déposés par excès de charge lors de la diminution de la vitesse de transport. L'énergie du courant a diminué progressivement avec accumulation des éléments de plus en plus fins et la précipitation chimique du calcaire étant favorisée en milieu marin sous faible énergie. La couleur noire des argiles est le témoin d'un dépôt dans des conditions calmes et réductrices ou anoxiques, elle peut indiquer également sa richesse en matière organique (Chevillon, 1992 ; Massala 1993). La couleur rougeâtre est la conséquence caractéristique de la mobilisation du fer dans un milieu plutôt oxydant (Bourkhir et al, 2017).

La présence de pyrite, de débris carbonés et de paillettes de micas témoignent des conditions réductrices du milieu de sédimentation où se sont accumulés ces dépôts (Taylor et Curtis, 1995). Cette association de pyrite, de glauconite et de débris carbonés montre un milieu de dépôt marin peu profond mais peu oxygéné avec un ralentissement de la sédimentation (Odin, 1975 ; Yao et al., 2011). La coexistence de la pyrite et de la glauconite dans un même milieu indique que la pyrite est postérieure à la glauconite (Yao, 2012). Selon Assalé (2013), la présence de débris carbonés et de la pyrite, dans les argiles noires, indique que celles-ci ont été déposées dans un milieu marin marginal pauvre en oxygène. Comme la pyrite ne peut se former qu'en milieu réducteur, il est fort probable que la glauconite soit héritée d'un milieu oxydant, donc moins réducteur (Yao, 2012). Par ailleurs, l'abondance de débris carbonés et de paillettes de micas, observée dans la présente étude, indique une sédimentation influencée par le continent. 


\section{- Granulométrie}

Les paramètres granulométriques indiquent que les sables sont très grossiers à moyens, assez bien à médiocrement classés, asymétriques vers les éléments grossiers et fins. Les courbes granulométriques ainsi que les courbes de fréquences révèlent la forme en $\mathrm{S}$ et un mode plurimodal qui confirment les résultats d'un faciès de sables littoraux bien classés avec évacuation de particules fines (Moiola et Weiser, 1968 ; Rivière, 1977 ; Pinot, 1994; Saidi et al., 2004). Les courbes cumulatives pour les sédiments des deux localités (Fresco et Grand-Lahou) ont, en général, la forme d'un « $\mathrm{S} »$; ceci témoigne d'un tri moyen des sédiments et d'une dynamique plus ou moins homogène, avec intervention à la fois de l'hydrodynamisme et d'une dynamique éolienne. La polymodalité de certaines courbes granulométriques simples témoigne de l'hétérogénéité des échantillons, les sédiments d'origine fluviatile proviendraient de plusieurs sources.

\section{- Morphoscopie}

L'observation morphoscopique des grains de quartz, à la loupe binoculaire, a permis de mettre en évidence une abondance de grains émoussés luisants. L'abondance de cette famille de grains indique un mode de transport aquatique (Vatan A., 1967 ; Kra, 2016).

\section{- Calcimétrie}

Les très faibles taux de carbonates observés dans certains intervalles des sondages (Fresco) peuvent, comme l'indique les données lithologiques, suggérer un milieu agité, défavorable aux dépôts de carbonates. Selon les travaux de Selley (1978), Yao et al. (2011), Gbangbot et al. (2012), les faibles teneurs de carbonates $\left(\mathrm{CaCO}_{3}\right)$ traduisent un environnement de forte énergie à Fresco et un régime hydrodynamique faible à Grand-Lahou, où les taux de $\mathrm{CaCO}_{3}$ sont plus élevés. L'abondance des coquilles entières témoigne d'une dynamique de dépôt moyenne à faible. Les dépôts de Grand-Lahou paraissent être déposés sous l'action d'une dynamique relativement faible dans un milieu peu profond.

\section{Conclusion}

L'approche lithostratigraphique des localités de Fresco et de GrandLahou montre que cette zone est dominée par le faciès sableux et le faciès argileux et suggère un milieu de dépôt soumis à un hydrodynamisme contrasté. Les paramètres granulométriques montrent que ces sables sont très grossiers à moyens, assez bien à mal classés, asymétriques vers les éléments fins. Les courbes granulométriques simples ainsi que les courbes de fréquences révèlent un mode plurimodal et une forme en $\langle S »$, ceci témoigne d'une dynamique plus ou moins homogène et d'une hétérogénéité des sédiments. L'observation 
morphoscopique montre une dominance des grains émoussés luisants indiquant un mode de transport aquatique plus ou moins prolongée. L'analyse calcimétrique révèle un taux de carbonates élevé à Grand-Lahou, où les sédiments s'accumulent dans des milieux calmes ou lors de la diminution de la vitesse de transport par excès de charge.

Les résultats obtenus témoignent que les sédiments étudiés proviennent de plusieurs sources continentales sous influence fluviatile et se sont déposés dans des milieux peu profonds.

\section{Remerciements}

Les auteurs souhaiteraient adresser leurs remerciements à la Direction de la Société Africaine Hydraulique de Côte d'Ivoire qui a autorisé les prélèvements des échantillons ayant servi à la réalisation de ce travail. Les membres du Laboratoire LGTE de la Faculté des Sciences d'El Jadida sont remerciés pour leur soutien.

\section{References:}

1. Affian, K., 1986. Incidence réciproque des structures océaniques et continentales sur l'évolution des bassins ouest Africain (Golfe de Guinée et de la Côte d'Ivoire Méridionale).Thèse de 3ème cycle océanographique, Université, Bordeaux I, No 2167, 259 p.

2. Aka, K., 1991. La sédimentation quaternaire sur la marge de la Côte d'Ivoire: Essai de Modélisation. Thèse de Doctorat d'Etat des Sciences Naturelle, Université d'Abidjan, 146, 233p.

3. Assalé, F.Y. P., 2013. Caractérisation sédimentologique, palynologique, géochimique et paléoenvironnementale des formations sédimentaires connexes à la faille des lagunes (Est du bassin onshore de Côte d'Ivoire). Thèse de doctorat, Université Félix Houphouët Boigny Cocody 361p.

4. Bacchiana, C., 1982. L’étude de la région Abidjan, Anyama, Alépé, Bassam. Ministère des Mine DH., 110p.

5. Bamba, K.M., Digbehi Z.B., Sombo, B.C., Goua, E.T. et N'da, V.L., 2011: Foraminifère planctoniques, biostratigraphique et paléoenvironnement des dépôts Albo-Turoniens de la Côte d'Ivoire, Afrique de l'Ouest. Revue de Paléobiologie, 30, 1-11.

6. Boukhir, M., Jamal, M et Azzouzi K., 2017: Étude Lithostratigraphique Des Formations

7. Superficielles Urbaines Du Secteur Nord De La Ville De Fès, Maroc. European Scientific Journal., 13, n³3, 129 p.

8. Cailleux, A., 1947. Distinction des sables marins et fluviatiles. Bulletin. Revue Société Géologique France, $5^{\text {ème }}$ série, t. xv, 375-404. 
9. Cailleux, A. et Tricart, J., 1959. Initiation à l'étude des sables et des galets. Centre de documentation universitaire, fascicules, Paris, 3, 202 p.

10. Chamley, H., 1988. Les milieux de sédimentation. Edition Lavoisier et Bureau de Recherches Géologiques et Minier (BRGM), Paris \& Orléans, $168 \mathrm{p}$.

11. Chevillon, C., 1992. Biosédimentologie du grand lagon Nord, Etude et Thèse, ORSTOM, Paris, 223p.

12. Cremer, M., 1983. Approches sédimentologiques et géophysiques des accumulations turbiditiques. L'éventail profond du Cap-Ferret (Golfe de Gascogne), la série des grès d'Annot (Alpes de haute province). Thèse Doctorat d'Etat Es-Sciences., Université. Bordeaux I (France), 344p.

13. Defaflia, N., Abdessadok. S., Djerrab. A et Chouabbi. A., 2013. Etude Sédimentologique et Paléoenvironnementales des Formations Quaternaires Alluvionnaires De L'oued Birzguel, El Ma El Abiod, Wilaya De Tebessa. Algérie Université Mohamed Khider - Biskra, Algérie, Courrier du Savoir, 17, 139-147.

14. Digbehi, Z.B., 1987. Etude compare de la sédimentation de premiers stades d'ouverture de l'Atlantique: Golfe de Guinée-Golfe de Gascogne. Thèse de Doctorat d'Etat Es-Sciences. Université. Pau et des pays de l'Adour, $229 \mathrm{p}$.

15. Digbehi, Z.B., N'da, L.V., Yao, K. R. et Atteba, Y. A. 1997. Principaux foraminifères et palynomorphes crétacés du bassin sédimentaire de Côte d'Ivoire, Golfe de Guinée septentrional: propositions pour une échelle biostratigraphique locale. Africa Geoscience Review, 4, 461-473.

16. Flores, R. M., Ethridge, F. G., Miall, A. D., Galloway, W. E and Fouch, T. D., 1985. Recongnition of fluvial depositional systems and their resource potential. SEPM special publication.

17. Gbangbot, J-M. K., 2012. Caractérisation stratigraphique des aquifères des formations de subsurface de la région des lagunes de côte d'ivoire : essai de modélisation des environnements de dépôts du tertiaire. Thèse de doctorat, Université. Félix Houphouët Boigny. Cocody, $196 \mathrm{p}$.

18. Hinschberger, F.et Pomel, R., 1979. Oro-hydrographie. In : Atlas de Côte-d'Ivoire. Ministère Plan/ORSTOM/ Institut Géographie Tropicale Abidjan.

19. Kouassi, A. K., Yao N. J. P., Bié G. R., Digbehi, Z. B., Bamba M. K., Goua, T. E. et Yao, K. C., 2013. Essai de caractérisation micropaléontologique et paléo-environnementale et mise en évidence de l'EAO2 à l'interface cénomanien/turonien $(\mathrm{C} / \mathrm{T})$ dans le bassin 
sédimentaire de côte d'ivoire, Afrique de l'ouest, Revue Ivoirienne des Sciences et Technologie., 21/22 (2013) 95-118.

20. Kra, A. C., 2016. Exoscopie des quartz pour la reconstitution paléogéographique des formations de l'est du bassin onshore de la côte d'ivoire : cas des localités d'Anyama et de Bonoua. Thèse de Doctorat, Université Félix Houphouët Boigny. Cocody, 265p.

21. Lowe, D., 1982. Sediment garvity flows II-depositional models with special reference to the deposits of high-density turbidity current. Journal of Sedimentary Petrology., 52, n 1, 279-297.

22. Massala, A., 1993. Le Crétacé supérieur et le Tertiaire du bassin côtier congolais. Biochronologie et stratigraphie séquentielle. Thèse de Doctorat, Université de Brazaville, 250p.

23. Moiola R.J. et Weiser D., (1968). Textureal parameters: an evaluation. Journal Sedimentary Petrology, 38, 45-53.

24. Miall, A. D., 1996. The geology of fluvial deposits. Sedimentary facies, Basin analysis and petroleum Geology .Springer $582 \mathrm{p}$

25. Odin, G.S., 1975. Les glauconies: constitution, formation, âge. Thèse de Doctorat, Université Paris-Ronéo, France, 250 P.

26. Petit-john, F.J., 1957. Sedimentary rocks. Ed. Harper and Brothers, New-York., 2nd Edition, 718 p.

27. Pinot, J.P., 1994. Manipulations sédimentologiques courantes. Edition 07 Février. Sedimentology, 118p.

28. Rivière, A., 1977. Méthodes granulométriques, techniques et interprétations. Ed. Masson, New York, Paris, Barcelone, Milan 149p.

29. Saidi, H., Brahim, M. et Gueddari M., 2004. Caractérisation granulométrique et minéralogique des sédiments de surface de la frange littorale Sidi Bou Said-la Goulette, Mer de Salammalô, Tunisie. Bulletin de l'Institut National des Sciences et Techniques, 31, 97-106.

30. Saint Marc, P. et N'da, Loukou., 1997: Biostratigraphie et environnements des dépôts crétacés au large d'Abidjan (Golfe de Guinée). Cretaceous research, 18,545-565

31. Soro, T D., Soro N., Oga, Y M-S., Lasm, T., Soro, G., Ahoussi, K E et Biemi J., 2011. La variabilité climatique et son impact sur les ressources en eau dans le degré carré de Grand-Lahou (Sud-Ouest de la Côte d'Ivoire), Physiology and Geology, 5/1, 55-73.

32. Spengler, A et Delteil, J. R., 1966. Le bassin secondaire-tertiaire de Côte d'Ivoire (Afrique occidentale). In Reyre D - Bassins sédimentaires du littoral africain, Assistance Service Géologique Afrique., Paris, 99-113.

33. Selley, R.C., 1978. Ancient sedimentary environments. Second edition, 287p. 
34. Stril, J.M., 1987. Faciès, séquence sédimentaire et géométrie des corps sédimentaires du plateau continental ivoirien. DESS, 302p.

35. Tastet, J. P., 1979. Environnements sédimentaires et structuraux quaternaires du littoral du Golfe de Guinée (Côte d'Ivoire, Togo, Bénin). Thèse de Doctorat d'Etat Es-Sciences Naturelle; Université de Bordeaux I, 621, 175p.

36. Taylor, K. G. and Curtis. C. D., 1995. Stability and facies association of early diagenetic mineral assemblages; an example from a Jurassic ironstone-mudstone succession, U.K. Journal of Sedimentary Research, 65, 358-368.

37. Vatan, A., 1967. Manuel de sédimentologie. Edition Technip, Paris, Cedex 15,359, $399 \mathrm{p}$.

38. Vigreux, T., Aoustin, D. et Flotte, P., 2011. Enregistrement sédimentaire et environnement holocène de la plaine alluviale du Giessen (Scherwiller, Bas-Rhin, Alsace). Quaternaire, 22, (2), 2011, 129-145.

39. Yao, N. J-P., Digbehi, Z.B., Monde, S., Kra, A. C., Aka, K., Bleoue, N. Z., Tea, Y.J., Kplohi, Y.L.H et Duffi, K. L., 2011. Etude Sédimentologique et Esquisse Paléoenvironnementale des Formations de Fresco. Sciences \& Nature, 8(1-2), 73-84

40. Yao, N.J-P., 2012. Caractérisation Sédimentologique, minéralogique, géochimique et biostratigraphique des falaises vives de Fresco : région de Grand-Lahou (Côte d'Ivoire). Thèse de Doctorat, Université Félix Houphouët Boigny, Cocody (Côte d'Ivoire): 187 p 